\title{
Engineering of Aspergillus niger for the production of secondary metabolites
}

\author{
Lennart Richter ${ }^{1 \dagger}$, Franziska Wanka ${ }^{2 \dagger}$, Simon Boecker ${ }^{1,2}$, Dirk Storm² ${ }^{2}$, Tutku Kurt ${ }^{2}$, Özlem Vural ${ }^{2}$, \\ Roderich Süßmuth ${ }^{{ }^{*}}$ and Vera Meyer ${ }^{2^{*}}$
}

\begin{abstract}
Background: Filamentous fungi can each produce dozens of secondary metabolites which are attractive as therapeutics, drugs, antimicrobials, flavour compounds and other high-value chemicals. Furthermore, they can be used as an expression system for eukaryotic proteins. Application of most fungal secondary metabolites is, however, so far hampered by the lack of suitable fermentation protocols for the producing strain and/or by low product titers. To overcome these limitations, we report here the engineering of the industrial fungus Aspergillus niger to produce high titers (up to $4,500 \mathrm{mg} \cdot \mathrm{I}^{-1}$ ) of secondary metabolites belonging to the class of nonribosomal peptides.

Results: For a proof-of-concept study, we heterologously expressed the $351 \mathrm{kDa}$ nonribosomal peptide synthetase ESYN from Fusarium oxysporum in A. niger. ESYN catalyzes the formation of cyclic depsipeptides of the enniatin family, which exhibit antimicrobial, antiviral and anticancer activities. The encoding gene esyn 1 was put under control of a tunable bacterial-fungal hybrid promoter (Tet-on) which was switched on during early-exponential growth phase of $A$. niger cultures. The enniatins were isolated and purified by means of reverse phase chromatography and their identity and purity proven by tandem MS, NMR spectroscopy and X-ray crystallography. The initial yields of $1 \mathrm{mg} \cdot \mathrm{I}^{-1}$ of enniatin were increased about 950 fold by optimizing feeding conditions and the morphology of $A$. niger in liquid shake flask cultures. Further yield optimization (about 4.5 fold) was accomplished by cultivating A. niger in 5 I fed batch fermentations. Finally, an autonomous A. niger expression host was established, which was independent from feeding with the enniatin precursor D-2-hydroxyvaleric acid D-Hiv. This was achieved by constitutively expressing a fungal D-Hiv dehydrogenase in the esyn 1-expressing A. niger strain, which used the intracellular a-ketovaleric acid pool to generate D-Hiv.

Conclusions: This is the first report demonstrating that $A$. niger is a potent and promising expression host for nonribosomal peptides with titers high enough to become industrially attractive. Application of the Tet-on system in A. niger allows precise control on the timing of product formation, thereby ensuring high yields and purity of the peptides produced.
\end{abstract}

Keywords: Aspergillus niger, Secondary metabolite, Nonribosomal peptide synthetase, Enniatin, Heterologous gene expression

\footnotetext{
* Correspondence: suessmuth@chem.tu-berlin.de; vera.meyer@tu-berlin.de

${ }^{\dagger}$ Equal contributors

'Institute of Chemistry, Department of Biological Chemistry, Berlin University

of Technology, Straße des 17, Juni 124, 10623 Berlin, Germany

${ }^{2}$ Institute of Biotechnology, Department Applied and Molecular

Microbiology, Berlin University of Technology, Gustav-Meyer-Allee 25, 13355

Berlin, Germany
}

\section{Biomed Central}

(c) 2014 Richter et al.; licensee BioMed Central Ltd. This is an Open Access article distributed under the terms of the Creative Commons Attribution License (http://creativecommons.org/licenses/by/4.0), which permits unrestricted use, distribution, and reproduction in any medium, provided the original work is properly credited. The Creative Commons Public Domain Dedication waiver (http://creativecommons.org/publicdomain/zero/1.0/) applies to the data made available in this article unless otherwise stated. 


\section{Background}

Recent genome mining efforts have uncovered that the genomes of filamentous fungi encode an unexpected rich repertoire of low-molecular-weight compounds with commercial relevance. These natural products known as secondary metabolites include nonribosomal peptides, polyketides and lipopeptides, which have pharmacological implications. Isoprenoids are interesting for the food industry as nutraceuticals or aroma compounds and poly-unsaturated fatty acids or lipids, can potentially be commercialized as biofuels. The natural product portfolio of filamentous fungi is thus impressive and emphasizes their great potential to become multi-purpose expression platforms in biotechnology. However, most of the genes involved in secondary metabolism pathways are not expressed under standard laboratory or industrial conditions and/or are present in intractable filamentous fungi, which prevents application of these natural products [1-3]. Different strategies based on molecular and epigenetics factors as well as cultivation methods have thus been undertaken to awaken these silent genes $[4,5]$. In brief, secondary metabolite (SM) production is under control of complex regulatory gene networks and involves intricate multi-step biosynthetic machineries, as well as major reorganization of primary metabolic fluxes to redirect cellular metabolic resources towards their biosynthesis. SM expression is naturally linked with starvation-induced developmental processes leading to (a)sexual spore formation [6-8]. These processes can easily be tracked and even induced during bioreactor cultivations by adjusting low growth rates $[9,10]$.

The advent of synthetic biology opens new avenues to express any SM gene of interest in a filamentous fungal host which is easily tractable by genetic engineering. For example, the geodin gene cluster of Aspergillus terreus was recently reconstituted in $A$. nidulans and the penicillin cluster of $P$. chrysogenum was completely rewired and expressed from a polycistronic gene cluster under control of a single xylose-inducible promoter in $A$. nidulans [11-13]. Another system for A. nidulans is based on expression of any fungal SM gene of interest under control of an alcohol-inducible promoter and includes methods for deletion entire $A$. nidulans SM gene clusters. This approach is especially interesting as it eliminates production of the most abundant $A$. nidulans SMs, thus reducing the SM background and facilitating purification of the heterologously expressed SMs [14]. None of the inducible promoters used so far is tunable, carbon source-independent and tight under non-induced conditions. This, however, poses limitations in their use, especially when the switch in the carbon source affects changes in the primary metabolic fluxes which should provide precursors for heterologous SM production. This limitation, however, can be overcome by applying an artificial expression system based on the Tet-on system, which was established and systematically evaluated for use in $A$. niger but is functional in many other filamentous fungi [15-17]. The Tet-on system is a tunable bacterial-fungal hybrid expression system, which becomes induced upon addition of the tetracyclinderivative doxycycline (Dox). Importantly, the inducing power depends on the Dox concentration applied and reaches expression levels which can compete with the strength of one of the strongest promoters known for filamentous fungi, the gpdA promoter from the glycolytic pathway [15].

We chose the industrial fungus A. niger as the expression host to determine whether or not the Tet-on system can be applied to produce high amounts of fungal SMs. Although the genome of $A$. niger carries the pptA gene encoding the key biosynthetic enzyme of fungal SM pathways (a 4'-phosphopantetheinyl transferase responsible for posttranslational activation of nonribosomal peptide synthetases and polyketide synthetase [18]), $A$. niger has so far only been exploited as expression platform for large-scale production of organic acids, proteins and enzymes [19]. Most importantly, the Tet-on system allows free choice over the timing of product formation as it can be switched on at any time during cultivation of $A$. niger [15]. We decided to induce heterologous SM expression during the exponential growth phase of $A$. niger due to two reasons. First, a maximum of ATP and primary metabolism intermediates are available during exponential growth phase, hence very high SM yields could supposedly be achievable. Second, endogenous SMs of $A$. niger become mainly expressed during carbon-starvation, i.e. during post-exponential growth phase $[9,10,20]$. Hence, heterologous SM production can be decoupled from homologous SM production and the $A$. niger cultures could largely be kept SM background-free.

For the proof-of-concept study, we decided to express the enniatin synthetase ESYN from Fusarium oxysporum in $A$. niger. Enniatin is a mixture of nonribosomal peptides and belong to the group of cyclic depsipeptides [21] which are mainly produced by the genus Fusarium (for reviews see $[22,23])$. Enniatin is synthesized by the multifunctional enzyme ESYN, which uses three D-hydroxycarboxylic acids and three L-amino acids as precursors and requires the cofactors ATP and $S$-adenosylmethionine (Figure 1, $[24,25])$. ESYNs from various Fusarium species use different amino acid precursors and display relaxed substrate specificities, which results in a wide spectrum of naturally occurring enniatins (Figure 2). After the first isolation of enniatin in 1947 [26], at least 29 naturally occurring derivatives were isolated from Fusaria.

Enniatin features antimicrobial [27], antiviral [28], cytotoxic [29] and phytotoxic [30] effects. For example, 


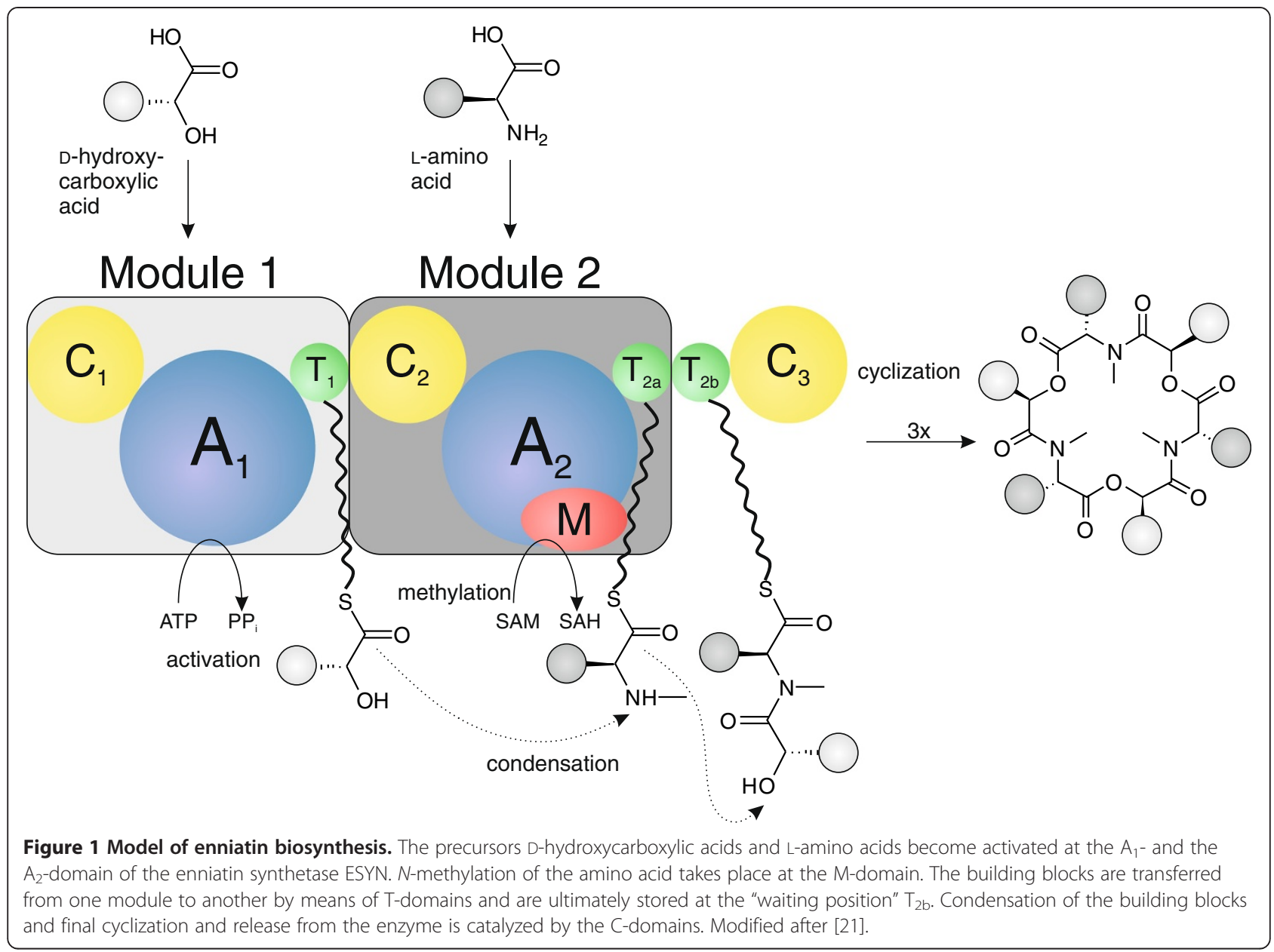

fusafungin, which is a mix of three derivatives (enniatin A, B and C), is a bactericide acting against grampositive and gram-negative bacteria and is used as a topical agent for the treatment of respiratory infections [31]. The mode of action of enniatin is mainly linked to its ionophoric activity. It is known that enniatin B forms complexes with cations in the ratio $1: 1,2: 1$ or 2:3 and complexes $\mathrm{K}^{+}, \mathrm{Ca}^{2+}, \mathrm{Na}^{+}, \mathrm{Mg}^{2+}$ and $\mathrm{Li}^{+}$[32], thereby forming cation-selective pores in biomembranes $[33,34]$. Additionally, the bioactivity of enniatins can be linked to their inhibition of drug efflux pumps [35] and cholesterol acyltransferase activity [36].

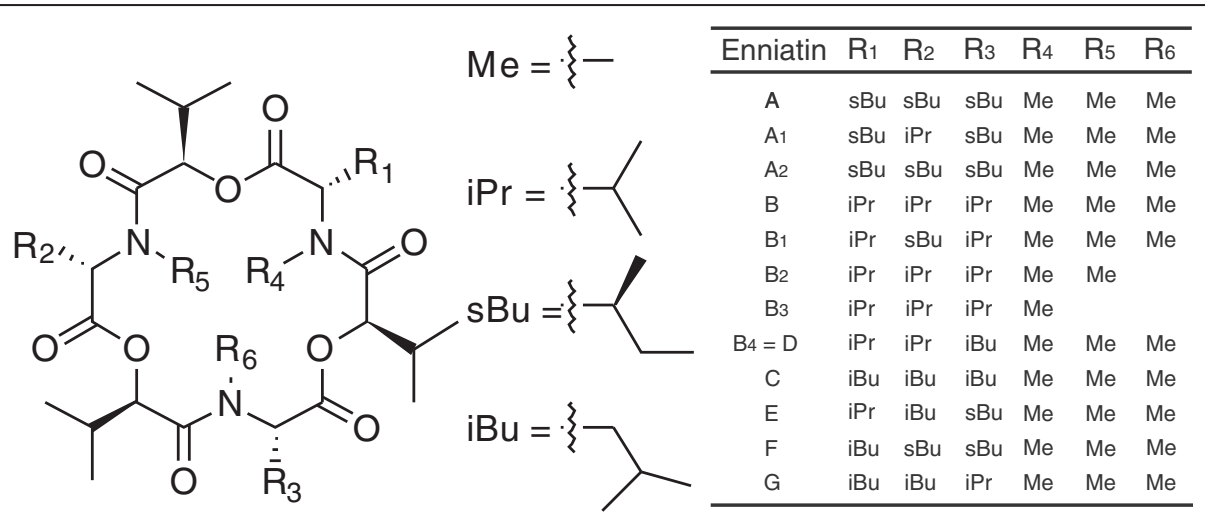

Figure 2 Amino acid composition and methylation pattern of the enniatin family. Enniatins are composed of three $D$-hydroxycarboxylic acids and three $L$-amino acids. The structural diversity is defined by the incorporation of different $L$-amino acids $\left(R_{1}-R_{3}\right)$, which can be valine (iPr), leucine (iBu) or isoleucine (sBu). L-amino acids can be methylated (Me, $\mathrm{R}_{4}-\mathrm{R}_{6}$ ). Modified after [22]. 
The F. oxysporum enniatin synthetase ESYN synthesizes enniatin by coupling three D-hydroxycarboxylic acids and three L-amino acids via amide and ester bonds in an alternating fashion (Figure 2). Whereas ESYN can accept different amino acids as precursors (L-valine, L-isoleucine or L-leucine), only one species of D-hydroxycarboxylic acid can be found in natural enniatin isolates (D-hydroxyisovaleric acid, D-Hiv). D-Hiv stems from the L-valine metabolism: L-valine is deanimated by a valine aminotransferase to 2-keto-isovaleric acid (2-Kiv), which becomes subsequently reduced by a keto-isovaleric acid reductase (KivR) under consumption of $\mathrm{NAD}(\mathrm{P}) \mathrm{H}$ to D-Hiv. In contrast to $F$. oxysporum, $A$. niger has not been reported so far to produce enniatin. Although open reading frames with weak similarity to the esyn 1 gene of $F$. oxysporum are present in its genome (An01g11770, An08g02300, An11g00050, An12g07230, An13g03040, [20]), it lacks a kivR gene [20]. Hence, $A$. niger could potentially be able to produce enniatin, given that D-Hiv is present in the medium.

The main objective of this study was to determine whether $A$. niger is a suitable expression host for highlevel production of fungal nonribosomal peptides. We therefore put the esyn 1 gene from $F$. oxysporum under control of the Dox-inducible Tet-on system and expressed it heterologously in $A$. niger to produce the enniatin as a model nonribosomal peptide. We optimized the production conditions using a design-of-experiment (DOE) approach which addressed medium composition, D-Hiv feeding conditions and Dox concentration. We furthermore optimized the cultivation conditions by establishing batch and fed batch cultures for an esyn 1 expressing $A$. niger strain. Finally, we engineered an autonomous enniatin $\mathrm{B}$ producing $A$. niger strain which is independent from D-Hiv feeding.

\section{Results}

Heterologous expression of the esyn 1 gene in A. niger

The esyn1 gene of $F$. oxysporum was integrated in plasmid pVG2.2 [15] to give plasmid pDS4.2, which comprises all three components of the Tet-on system: $\operatorname{PgpdA}:: r t T A 2^{S}-M 2$ for constitutive expression of the transactivator rtTA, tetO7::Pmin::esyn1, which mediates esyn1 expression in a Dox-dependent manner (note that rtTA is only able to bind to its operator sequence tetO7 when bound to Dox, [15]) and the pyrG* cassette, necessary for selection and targeting of the system to the pyrG locus of $A$. niger (Additional file 1: Figure S1). As recipient strain, the protease-negative ( $p r t T^{-}$) and uracil-auxotroph (pyrG ${ }^{-}$) strain AB1.13 [37] was used. Uridine-prototroph transformants were selected and screened via PCR and Southern analysis for the presence of single or multiple pDS4.2 copies in the genome of $A$. niger (Additional file 1: Figure S1 and data not shown). Ten pDS4.2-carrying transformants were selected and cultivated in liquid shake flask cultures in the presence or absence of Dox. Controls were an $A$. niger wild type strain (strain N402), the original producer F. oxysporum (strain ETH1536) and an A. niger strain harboring a single copy of the esyn1-free plasmid pVG2.2 at the pyrG locus (strain VG5.1). After cultivation, enniatin was isolated from the biomass and the culture supernatant by means of ethyl acetate extraction. The amount of enniatin produced was determined and quantified by HPLC-MS. Among the transformants, strain DS3.1, which carried a single copy of pDS4.2 at the pyrG locus, produced the highest amount of enniatin (about $1 \mathrm{mg} \cdot \mathrm{l}^{-1}$, Figure 3). Only minute amounts of enniatin were detectable in the control strains N402 and VG5.1 and all pDS4.2 carrying strains in the absence of Dox, verifying that the expression system is tight under noninduced conditions. The $\mathrm{m} / z$ values and retention time of enniatin isolated from the different $A$. niger transformants were equal to those extracted from the natural enniatin producer F. oxysporum (Figure 4 and data not shown). Several derivatives could be detected and characterized by tan$\operatorname{dem} \mathrm{MS}$, amongst them enniatin $\mathrm{A}, \mathrm{A}_{1}, \mathrm{~B}$ and $\mathrm{B}_{1}$ (data not shown). However, because the standard used was a mixture of enniatin $\mathrm{A}, \mathrm{B}$, and $\mathrm{C}$ isolated from $F$. oxysporum, the exact ratio and amount of the enniatin variants could not be determined. However, full MS-scans of the standard and DS3.1 samples showed that the enniatin composition was similar to F. oxysporum (Figure 4).

\section{Optimization of enniatin production}

In order to identify the optimum condition for high yield production of enniatin, a design-of-experiment approach was followed using the statistical software program MODDE (see Methods). The following parameters were varied in $20 \mathrm{ml}$ shake flask cultures of the esyn1expressing strain DS3.1: (i) medium composition (minimal medium, complete medium, Fusarium defined medium)

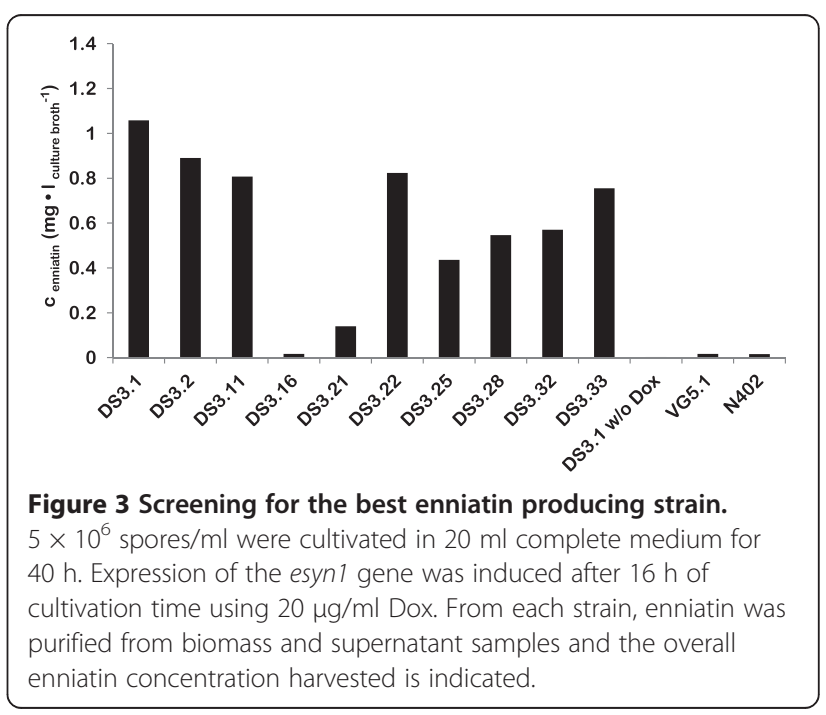




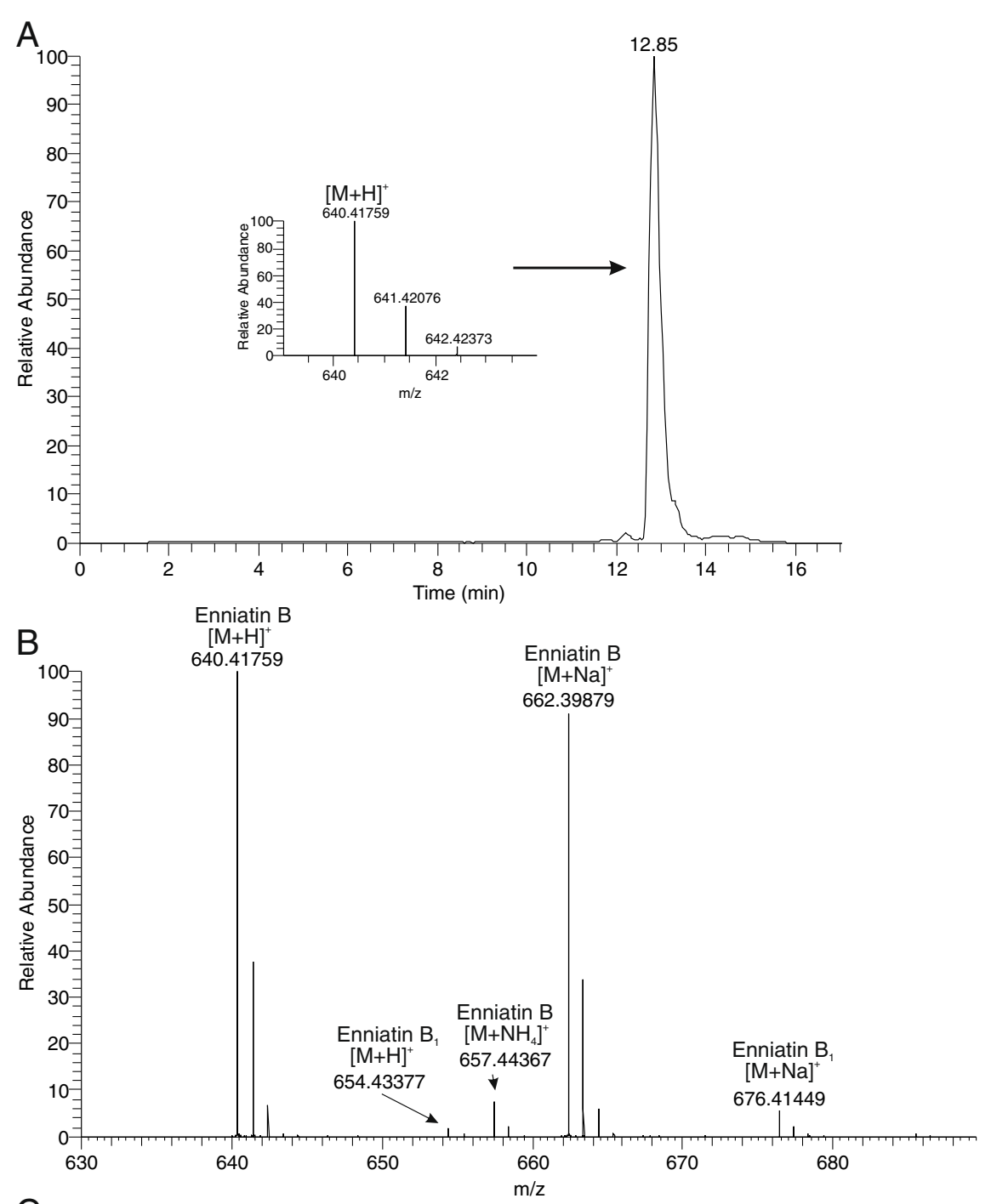

C

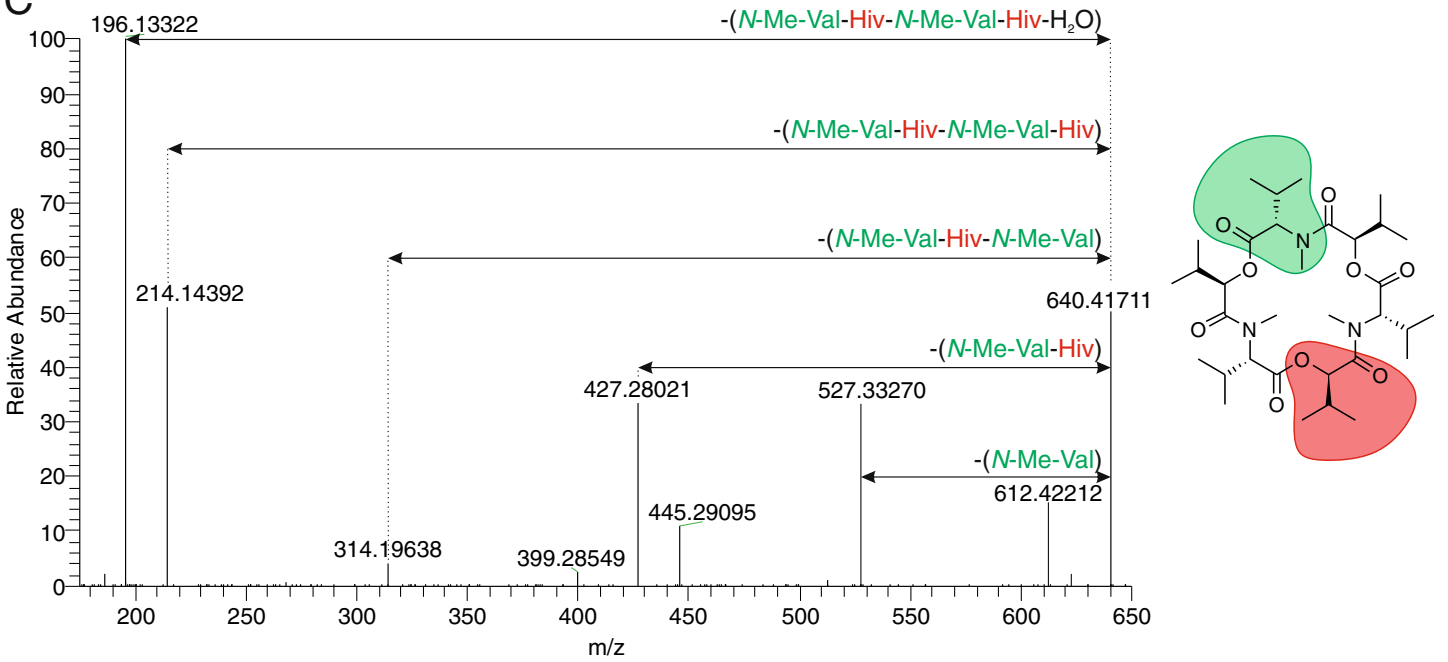

Figure 4 (See legend on next page.) 
(See figure on previous page.)

Figure 4 Analysis of enniatins produced by strain DS3.1. A) HR-LCMS chromatogram of purified enniatin B. A segment from the mass spectrum shows $\left[\mathrm{M}+\mathrm{H}^{+}\right]$of enniatin $\mathrm{B}$ with the characteristic isotope pattern. The main peak can be assigned to enniatin B. Minor impurities can be detected at retention time $11.8 \mathrm{~min}$ and $12.2 \mathrm{~min}$. B) HR-LCMS average mass spectrum. As example, the mass spectrum of purified enniatin B is shown. The $\mathrm{H}^{+}$, $\mathrm{NH}_{4}^{+}, \mathrm{Na}^{+}$adducts of enniatin B can be observed. The sample contains small amounts of enniatin B1. Samples were measured on an ESI-Orbitrap-MS. C) ESI-HRMS/MS spectrum obtained with a LTQ Orbitrap XL apparatus using direct injection and applying a collision energy of $12 \mathrm{eV}$. The moiety highlighted in green represents the L-valine and the moiety highlighted in red represents D-Hiv incorporated into the enniatin $B$ structure. For the fragments $\mathrm{m} / \mathrm{z}$ values were calculated. The calculated $\mathrm{m} / \mathrm{z}$ value for the $\left[\mathrm{C}_{27} \mathrm{H}_{47} \mathrm{~N}_{2} \mathrm{O}_{8}\right]^{+}$fragment is 527.33269 and the $\mathrm{m} / \mathrm{z}$ value observed was 527.33270. For the $\left[\mathrm{C}_{22} \mathrm{H}_{39} \mathrm{~N}_{2} \mathrm{O}_{6}\right]^{+}$fragment, the calculated $\mathrm{m} / \mathrm{z}$ value was 427.28026 and the $\mathrm{m} / \mathrm{z}$ value observed was 427.28021. The calculated $\mathrm{m} / z$ value for the $\left[\mathrm{C}_{16} \mathrm{H}_{28} \mathrm{NO}_{5}\right]^{+}$fragment was 314.19620 and the $\mathrm{m} / \mathrm{z}$ value measured was 314.19638 . The calculated $\mathrm{m} / \mathrm{z}$ value for the $\left[\mathrm{C}_{11} \mathrm{H}_{20} \mathrm{NO}_{3}\right]^{+}$fragment was 214.14377 and the $\mathrm{m} / \mathrm{z}$ value observed was 214.14392 .

[38], (ii) L-valine, L-leucine, L-isoleucine supplementation (0, 10, $20 \mathrm{mM})$, (iii) D-Hiv supplementation $(0,5,10$, $50 \mathrm{mM})$, (iv) glucose concentration (1, 2.5, 5\%), (v) temperature $\left(26^{\circ} \mathrm{C}, 30^{\circ} \mathrm{C}\right)$, (vi) cultivation time $(24,36,48$, $92 \mathrm{~h})$ and (vii) Dox concentration $(0,5,10,20 \mu \mathrm{g} / \mathrm{ml})$. The parameters which mainly affected enniatin yields were Dox and D-Hiv (data not shown) and the best cultivation medium identified contained $20 \mathrm{mM}$ D-Hiv, $20 \mathrm{mM}$ of one of the amino acids and $10 \mu \mathrm{g} / \mathrm{ml}$ Dox. This medium composition improved the enniatin yield by a factor of 200 to $200 \mathrm{mg} \cdot \mathrm{l}^{-1}$ (Figure 5), whereby most of the enniatin could be extracted from biomass samples after $92 \mathrm{~h}$ of cultivation. Remarkably, the enniatin yield was further increased about 4.75-fold by increasing the glucose concentration to $5 \%$ and by adding talcum to the DS3.1 cultures (Figure 5). As reported recently, the addition of microparticles to liquid cultures of $A$. niger reduces the diameter of macromorphological pellets to only a few hundred micrometers. This in turn considerably improves uptake rates of nutrients and oxygen and increases the metabolic activity of A. niger [39]. Taken together, the final enniatin yield was $950 \mathrm{mg} \cdot \mathrm{l}^{-1}$ culture broth (corresponding to $0.04 \mathrm{~g} \cdot \mathrm{g}^{-1}$ dry weight biomass).

\section{Modulation of the enniatin product variety by targeted supplementation with amino acids}

In order to determine the scope of enniatin variants, which can be heterologously synthesized by $A$. niger, the precursor amino acids L-valine, L-leucine and L-isoleucine were added to DS3.1 cultures either individually or in combination. After cultivation for $72 \mathrm{~h}$, the profile of the synthesized enniatins was determined by HPLC-MS. When no or all three amino acids were added at the same concentration, the main products were enniatin $B$, $\mathrm{B} 1$ and B4, whereas enniatins $\mathrm{A}, \mathrm{A} 1, \mathrm{~A} 2, \mathrm{C}, \mathrm{E}$ and $\mathrm{F}$ were only present in trace amounts (Figure 6A). The latter fraction increased, when L-leucine or L-isoleucine were added and reached almost $50 \%$ of total enniatin, when both amino acids were supplemented each at $20 \mathrm{mM}$. When the cultivation medium was supplemented with L-valine only, the main product was enniatin B (87\%). Interestingly, enniatin variants containing
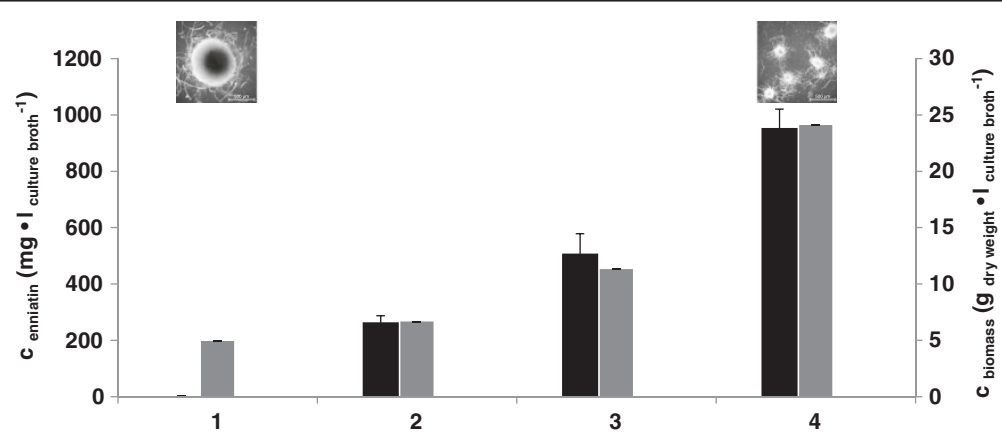

Figure 5 Optimization of the enniatin yield. $5 \times 10^{6}$ spores $/ \mathrm{ml}$ of strain DS3.1 were cultivated in $20 \mathrm{ml}$ shake flask cultures containing complete medium with varying composition. Induction of esyn 1 expression was performed in all media with $10 \mu \mathrm{g} / \mathrm{ml}$ Dox. Selected results are exemplarily shown: (1) $0 \mathrm{mM} \mathrm{L}$-valine/L-isoleucine/L-leucine, $1 \%$ glucose, $0 \mathrm{~g} / \mathrm{l}$ talcum, $40 \mathrm{~h}$ cultivation time, $30^{\circ} \mathrm{C}$ cultivation temperature. (2) $10 \mathrm{mM} \mathrm{L}$-valine/L-isoleucine/L-leucine, $1 \%$ glucose, $0 \mathrm{~g} / \mathrm{I}$ talcum, $10 \mathrm{mM}$ D-Hiv, $92 \mathrm{~h}$ cultivation time, $26^{\circ} \mathrm{C}$ cultivation temperature. (3) $10 \mathrm{mM}$ L-valine/L-isoleucine/L-leucine, $2.5 \%$ glucose, $2.5 \mathrm{~g} / \mathrm{l}$ talcum, $10 \mathrm{mM}$ D-Hiv, $92 \mathrm{~h}$ cultivation time, $26^{\circ} \mathrm{C}$ cultivation temperature. (4) $20 \mathrm{mM}$ L-valine/L-isoleucine/L-leucine, $5 \%$ glucose, $10 \mathrm{~g} / \mathrm{l}$ talcum, $10 \mathrm{mM}$ D-Hiv, $92 \mathrm{~h}$ cultivation time, $26^{\circ} \mathrm{C}$ cultivation temperature. The total enniatin concentration (black bars) and biomass concentration (grey bars) is given. Data from biological triplicates are shown. Microscopic pictures of DS3.1 pellets are shown. Bar, $500 \mu \mathrm{m}$. 
A

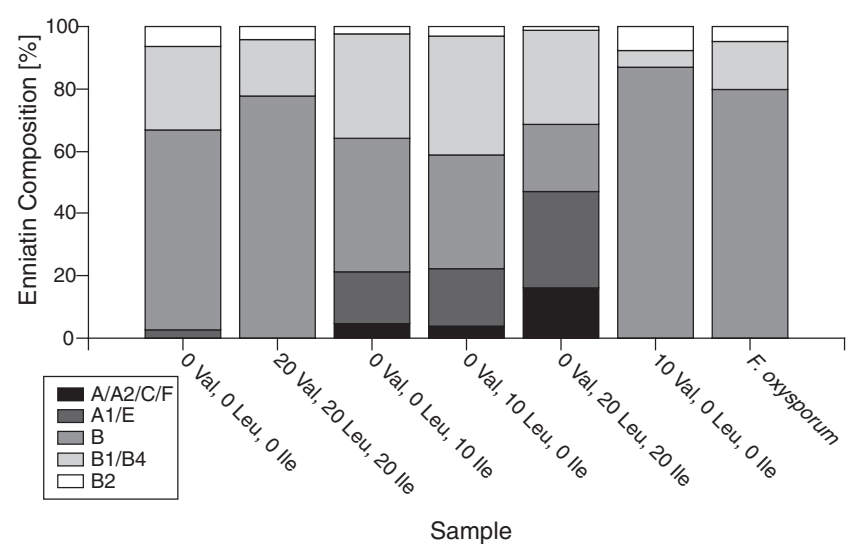

B

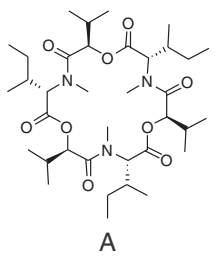

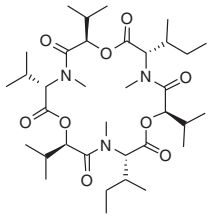

$\mathrm{A}_{1}$

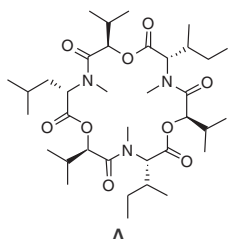

$\mathrm{A}_{2}$

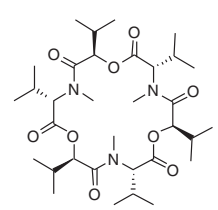

B

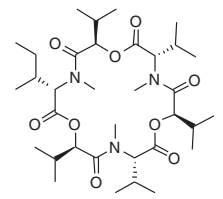

$\mathrm{B}_{1}$

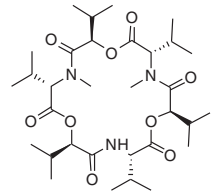

$\mathrm{B}_{2}$

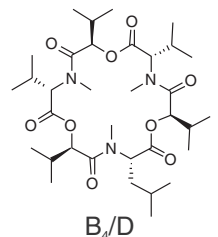

$\mathrm{B}_{4} / \mathrm{D}$
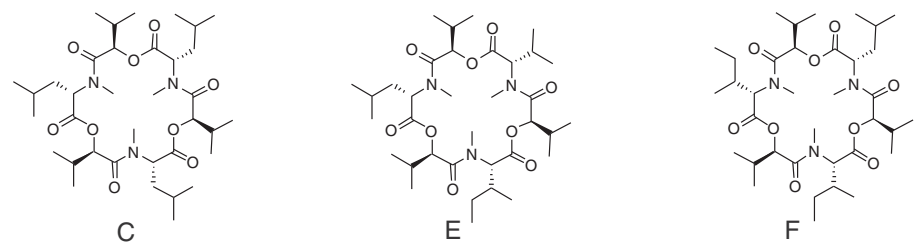

Figure 6 Spectrum of enniatin species produced by strain DS3.1. A) Different enniatin variants were produced depending on the amino acids fed. The final concentration of L-valine, L-leucine L-isoleucine in the cultivation medium is given in mM. B) Structures of enniatin species produced in strain DS3.1.

D-lactate moieties were also synthesized by strain DS3.1, which have so far not been observed in F. oxysporum. This suggests that the precursor repertoire which can be used by ESYN is broader in $A$. niger compared to F. oxysporum.

\section{Isolation and analytics of enniatin B}

The identity and purity of enniatin produced by strain DS3.1 was confirmed by ${ }^{1} \mathrm{H}-\mathrm{NMR}-,{ }^{13} \mathrm{C}-\mathrm{NMR}-$, IR-, MS-, MS/MS- and X-ray analysis (Additional file 2: Figure S2 and data not shown). From a 1 liter shake flask culture (20 mM D-Hiv, $20 \mathrm{mM} \mathrm{L-valine,} \mathrm{5 \%} \mathrm{glucose,} 10 \mu \mathrm{g} / \mathrm{ml}$ Dox, $10 \mathrm{~g} / \mathrm{l}$ talcum), $800 \mathrm{mg}$ enniatin could be isolated by crystallization, which corresponded to an enniatin yield of $0.04 \mathrm{~g} \cdot \mathrm{g}^{-1}$ dry weight biomass. The majority of enniatin was isolated from the biomass. The crystallized enniatin had a dark yellowish to brownish color and enniatin variats could be separated e.g. by means of preparative HPLC. After repetitive multiple crystallizations, a total of $675 \mathrm{mg}$ enniatin were obtained. The NMR spectra were identical to the ones published in literature [32,40] and proved that the crystals were pure enniatin B as summarized as follows: ${ }^{1} \mathrm{H}-\mathrm{NMR}$ spectrum: (400.1 $\mathrm{MHz}$, CDCl3) $\delta=5.11\left(\mathrm{~d},{ }^{3} J_{\mathrm{H}, \mathrm{H}}=8.7 \mathrm{~Hz}, 3 \mathrm{H}\right), 4.49\left(\mathrm{~d},{ }^{3} J_{\mathrm{H}, \mathrm{H}}=\right.$ $9.7 \mathrm{~Hz}, 3 \mathrm{H}), 3.11(\mathrm{~s}, 9 \mathrm{H}), 2.32-2.21(\mathrm{~m}, 6 \mathrm{H}), 1.05-$ $0.86 \mathrm{ppm}(\mathrm{m}, 36 \mathrm{H}) .{ }^{13} \mathrm{C}-\mathrm{NMR}$ spectrum: (100.6 $\mathrm{MHz}$, $\left.\mathrm{CDCl}_{3}\right) \delta=170.25,169.31,75.67,63.20,33.26,29.91$, 27.92, 20.42, 19.34, 18.72, 18.50 ppm. IR spectrum (Neat): $v=2963.6-2873.4\left(\mathrm{C}-\mathrm{H}, \mathrm{CH}_{3}\right.$ and $\left.\mathrm{CH}\right), 1736.1$ ( $\mathrm{C}=\mathrm{O}$, ester $), 1660.9(\mathrm{C}=\mathrm{O}$, amide $), 1183.6(\mathrm{C}-\mathrm{H}$, isopropyl) 1011.0 (CO, $\alpha$-hydroxycarboxylic acid). ESIHRMS spectrum: $m / z$ calculated for $\left[\mathrm{C}_{33} \mathrm{H}_{57} \mathrm{~N}_{3 \mathrm{O}}+\mathrm{Na}\right]^{+}$: 662.39870; found: 662.39859; ESI-HRMS/MS: $\mathrm{m} / z$ 
calculated for $\left[\mathrm{C}_{27} \mathrm{H}_{47} \mathrm{~N}_{2} \mathrm{O}_{8}\right]^{+}$: 527.33269; found: 527.33221, $m / z$ calculated for $\left[\mathrm{C}_{22} \mathrm{H}_{39} \mathrm{~N}_{2} \mathrm{O}_{6}\right]^{+}$: 427.28026; found: 427.27988, $m / z$ calculated for $\left[\mathrm{C}_{16} \mathrm{H}_{28} \mathrm{NO}_{5}\right]^{+}$: 314.19620; found: $314.19614, m / z$ calculated for $\left[\mathrm{C}_{11} \mathrm{H}_{20} \mathrm{NO}_{3}\right]^{+}$: 214.14377; found: 214.14375. The masses of the daughter ions are due to cleavages at the ester and amide bonds $(m / z=527.33,427.28,314.20,214.14)$ as described by [41].

The X-ray crystallographs demonstrated that the crystals had no impurities and were a complex of enniatin $B$ with $\mathrm{Na}^{+}$ions, whereby one $\mathrm{Na}^{+}$ion was located in the center of an enniatin B molecule (Additional file 2: Figure S2). As a result, the adjoining molecule from the next layer in the crystal is not located on the same axis but is shifted to the side. Thus, sandwich structures of enniatin $\mathrm{B}$ with the $\mathrm{Na}^{+}$ ions were not formed.

\section{Production of enniatin B by batch and fed batch bioreactor cultivation}

In order to obtain high enniatin yields under controlled conditions in bioreactors, $5 \mathrm{l}$ batch cultivations of strain DS3.1 were performed using a defined fermentation medium. This medium had a $\mathrm{pH}$ of 3 and was balanced as such, that glucose was the growth-limiting nutrient (final concentration $0.8 \%$; see Methods). Note that the low $\mathrm{pH}$ of the medium and the use of ammonia as nitrogen source ensures dispersed morphology of $A$. niger during bioreactor cultivation with no need for adding microparticles [42]. After the culture reached the early exponential growth phase (corresponding to $1 \mathrm{~g}$ biomass dry weight $\cdot \mathrm{l}^{-1}$ culture broth after about 14-16 $\mathrm{h}$ post inoculation), production of enniatin was induced by the addition of $10 \mu \mathrm{g} / \mathrm{ml}$ Dox, $20 \mathrm{mM}$ $\mathrm{D}-\mathrm{Hiv}$ and $20 \mathrm{mM}$ L-valine, respectively. In two independent runs, the maximal specific growth rate achieved was $0.24 \mathrm{~h}^{-1}$ and the biomass concentration peaked at $4.2 \mathrm{~g} \cdot \mathrm{kg}^{-1}$ culture broth after about $26 \mathrm{~h}$ post inoculation (Figure 7A). During exponential growth, pH 3 was maintained by the addition of $1 \mathrm{M}$ $\mathrm{NaOH}$, which has been shown to linearly correlate with the biomass accumulation and reflecting ammonium uptake during balanced growth $[9,43]$. The end of the exponential growth phase was detected by an increase of the dissolved oxygen signal (data not shown), after which the cell mass decreased by nearly $50 \%$ (Figure 7A). Importantly, the levels of $\mathrm{CO}_{2}$ and $\mathrm{O}_{2}$ in the exhaust gas clearly indicated that the cultures were still metabolically active, even 100 hours after depletion of the carbon source (data not shown). As recently demonstrated, carbon starvation of $A$. niger during submerged cultivation results in secondary growth by carbon recycling leading to a gradual transition from old to young hyphae [9]. Enniatin levels determined for selected time points demonstrated that enniatin B was mainly produced after carbon source depletion (i.e. after about $55 \mathrm{~h}$ post inoculation) and reached a maximum value of $0.29 \mathrm{~g} \cdot \mathrm{g}^{-1}$ dry weight biomass after about $110 \mathrm{~h}$ of cultivation (Figure 7A).

Finally, one fed batch cultivation was performed to increase biomass concentration and thereby enniatin B yield. After the culture reached the late exponential growth phase (corresponding to $4 \mathrm{~g}$ biomass $\cdot \mathrm{kg}^{-1}$ culture broth after about $18 \mathrm{~h}$ post inoculation), expression of the esyn 1 gene was induced by feeding with $5 \%$ glucose, $10 \mu \mathrm{g} / \mathrm{ml}$ Dox, $20 \mathrm{mM}$ D-Hiv and $20 \mathrm{mM} \mathrm{L}$-valine. To ensure that the esyn1 gene was continuously expressed at highest level, $10 \mu \mathrm{g} / \mathrm{ml}$ Dox were added every 4-7 h resulting in a final Dox concentration of $90 \mu \mathrm{g} / \mathrm{ml}$. As depicted in Figure 7B, this fermentation protocol ensured a specific growth rate of $0.15 \mathrm{~h}^{-1}$ and the biomass concentration reached $24.9 \mathrm{~g} \cdot \mathrm{kg}^{-1}$ culture broth after about $66 \mathrm{~h}$ post inoculation. Enniatin $B$ production started immediately after Dox induction and reached a maximum of $4.5 \mathrm{~g} \cdot \mathrm{kg}^{-1}$ culture broth after $66 \mathrm{~h}$ of cultivation (corresponding to $0.18 \mathrm{~g} \cdot \mathrm{g}^{-1}$ dry weight biomass, Figure 7B). Interestingly, linear accumulation of biomass in the fed batch run was paralleled by linear accumulation of enniatin B (Figure 7B), suggesting that the dynamics of enniatin $B$ production under the Tet-on system displays primary metabolite kinetics.

\section{Establishment of an autonomous $A$. niger production strain}

As mentioned above, $A$. niger cannot produce enniatin autonomously as its genome lacks the kivR gene encoding the D-Hiv generating enzyme $\alpha$-ketoisovalerate reductase. In order to engineer an autonomous enniatin production strain which is independent from D-Hiv feeding, strain DS3.1 was transformed with the kivR gene from F. oxysporum (E-KivR), which was put under control of the constitutive gpdA promoter (see Methods). Three transformants carrying multi-copy integrations of the PgpdA::kivR construct (strains ÖV4.3, ÖV4.10, ÖV4.11, data not shown) were analyzed for enniatin production using $20 \mathrm{ml}$ shake flask cultures. After induction with a final concentration of $10 \mu \mathrm{g} / \mathrm{ml}$ Dox and supplementation of $20 \mathrm{mM} \mathrm{L}$-valine, the enniatin yield in all three transformants reached $0.03 \mathrm{~g} \cdot \mathrm{g}^{-1}$ dry weight biomass after $92 \mathrm{~h}$ of cultivation (data not shown), which is about $75 \%$ of the enniatin yield of strain DS3.1 when fed with $20 \mathrm{mM}$ D-Hiv and $20 \mathrm{mM}$ L-valine.

\section{Discussion}

The fungal kingdom of approximately 1.5 million species exhibits a huge reservoir of secondary metabolites that span a broad variety of structurally and chemically diverse natural products. This reservoir has and will increasingly have a considerable potential impact on human welfare. The need to identify and produce novel bioactive fungal products goes far beyond antimicrobials and includes the 

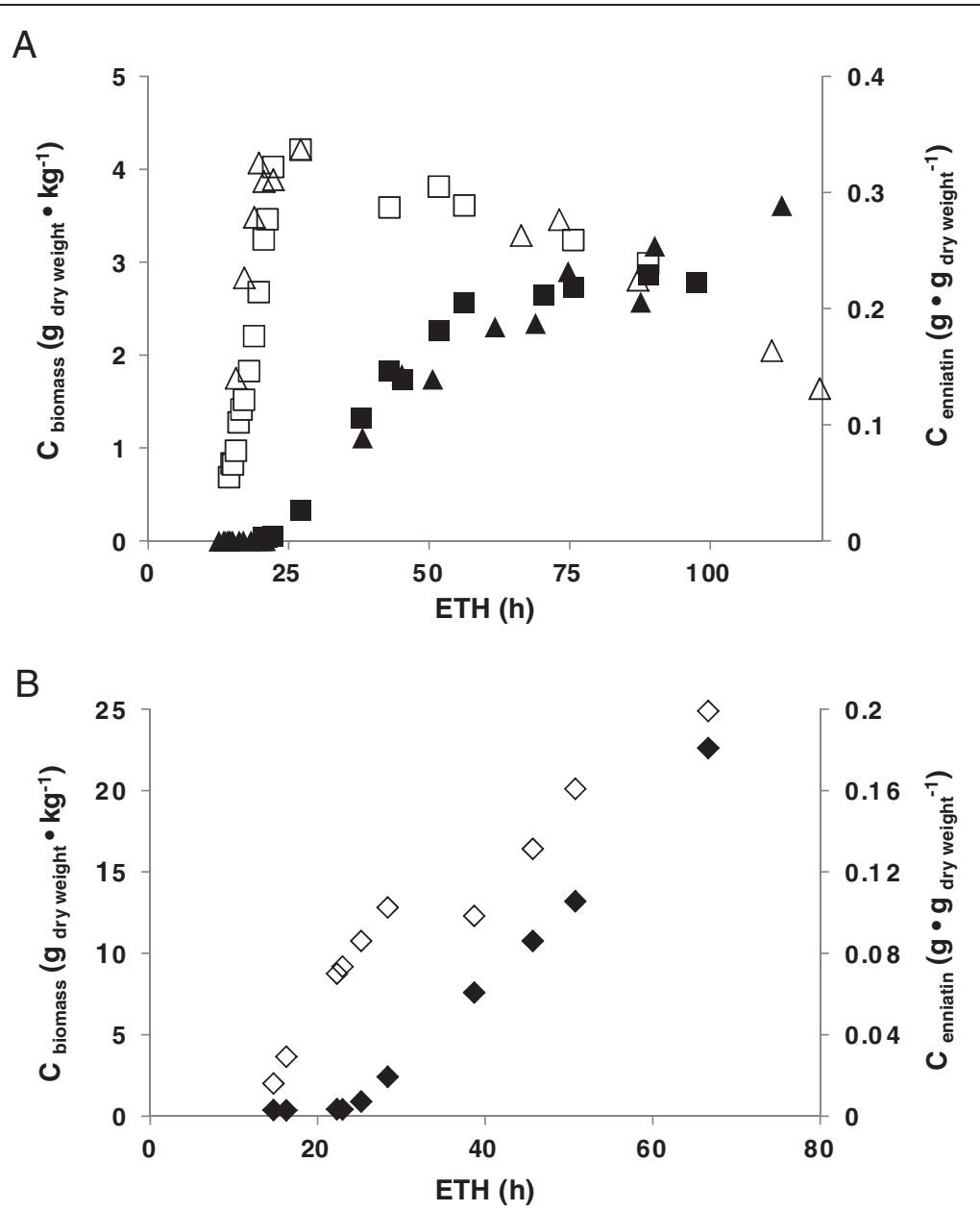

Figure 7 Submerged batch and fed batch cultivation of strain DS3.1. A) Biomass (open symbols) and enniatin accumulation (closed symbol) of two batch cultivations are shown. B) Biomass (open symbol) and enniatin accumulation (closed symbol) of a fed batch cultivation are shown.

requirement for novel drugs for various human health problems ranging from different cancers to neurodegenerative diseases, which are particularly emerging in aging societies. The newly emerged fungal 'omics' era and the advent of systems and synthetic biology provide innovative concepts and ideas to harness this untapped potential and to produce novel bioactive compounds from fungi on an industrial scale. The gene(s) or gene cluster(s) of interest can be of fungal or even (non)fungal origin and respective expression constructs can be plugged into fungal chassis strains allowing high expression levels. The gene(s) of interest will be expressed under the control of synthetic promoters and the product repertoire, the timing of product formation and productivity can be optimized using metabolic engineering strategies.

The aim of this study was to explore the potential of $A$. niger to become an expression system for secondary metabolites from other organisms. A. niger is so far being used in biotechnology as cell factory for the production of organic acids and secreted proteins [1]. For the proof-of-concept study, the cyclic depsipeptide enniatin was chosen. Heterologous production of cyclic depsipeptides such as beauvericin has been achieved in microbial host strains but only with very low yields, ranging from $3 \mathrm{mg} \cdot \mathrm{l}^{-1}$ in Escherichia coli to $100 \mathrm{mg} \cdot \mathrm{l}^{-1}$ in Saccharomyces cerevisiae $[44,45]$. Heterologous expression of the F. oxysporum esyn 1 gene in E. coli was accomplished as well; however, only minute amounts were produced ( $1 \mathrm{mg} \cdot \mathrm{l}^{-1}$; own unpublished data). Although chemical synthesis of enniatin is in principle possible, it can by far not fulfill the requirements for an efficient large-scale drug production process since its synthesis is too complex and very cost-intensive [46]. The pharmaceutical relevance of enniatin stimulated studies to use homologous fungal hosts such as $F$. sambucinum for enniatin production $([47,48])$. Although high amounts of enniatin were produced $\left(1.7 \mathrm{~g} \cdot \mathrm{l}^{-1}\right)$, these surface cultures lasted up to 5 weeks. Submerged shake flask cultivation of a randomly mutagenized $F$. oxysporum strain (strain ETH 1536) which included additional amino acid feeding 
resulted in the highest enniatin titer reported so far $\left(5 \mathrm{~g} \cdot \mathrm{l}^{-1}\right.$ after $96 \mathrm{~h}$ of cultivation, [38]).

Here, we demonstrate that heterologous expression of the esyn 1 gene under control of the Tet-on system in $A$. niger allows enniatin production rates which are considerably higher than ever reported for a heterologous host. $4.5 \mathrm{~g} \cdot \mathrm{l}^{-1}$ have been reached after $66 \mathrm{~h}$ of a fed batch cultivation of strain DS3.1, a yield which is sufficient for rapid scale-up, biological testing and commercial production. The yields which can be achieved with $A$. niger nearly reach the titer of the original production strain $F$. oxysporum. Two explanations might explain why A. niger is well suited for heterologous enniatin (and other nonribosomal peptides) production. First, A. niger possesses an endogenous PPTase, which is key for the posttranslational activation of NRPS [18]. Second, the ability to synthesize secondary metabolites is conserved in filamentous fungi. Their secondary metabolites are adaptive traits that have been subjected to natural selection during evolution. Although their occurrence apparently reflects particular life style and survival strategies and differ among fungal species, multiple secondary metabolic pathways are prevalent in each filamentous fungus. The existence of these pathways predestines filamentous fungi as hosts for heterologous fungal secondary metabolite production.

The genome of $A$. niger harbors five open reading frames (An01g11770, An08g02300, An11g00050, An12g07230, An13g03040), which display a weak similarity to the esyn1 gene of F. oxysporum [20]. Analysis of their expression profiles using published transcriptomics data from $A$. niger cultures subjected to carbon-limited growth in batch, chemostat and retentostat bioreactor fermentations $[9,49,50]$ revealed that An01g11770, An11g00050, An12g07230 and An13g03040 are silent under these conditions. An08g02300 though is expressed at low levels, with mean expression values of $10 \%$ or less when compared with the actin gene (An15g00560; data not shown). Hence, $A$. niger is likely to have the metabolic pathways and flexibility to synthesize enniatin with an esyn 1 gene being either of endogenous or exogenous origin. However, as it does not comprise the $k i v R$ gene encoding a D-Hiv dehydrogenase, feeding with D-Hiv or heterologous expression of a kivR gene is key to obtain high enniatin levels with $A$. niger as shown in this study. It has to be mentioned that An11g09950 shares high similarity with the D-Hiv dehydrogenase from Gibberella intermedi. An11g09950 is a predicted 2-dehydropantoate 2-reductase catalyzing a similar reaction as KivR, which is the reduction of 2-dehydropantoate to the D-hydroxycarboxylic acid D-pantoate under consumption of NADPH [50]. Due to the relatively high degree of similarity of both enzymes and their substrates, it might be conceivable that An11g09950 could accept $\alpha$-ketoisovalerate as substrate to synthesize D-Hiv. This would explain why enniatin is present in minute amounts in strain DS3.1 cultures when not fed with D-Hiv. In any case, establishment of an autonomous $A$. niger strain being independent of D-Hiv feeding will considerably reduce the cost of the fermentation process. Our data clearly demonstrated that heterologous expression of the kivR gene from F. oxysporum rendered $A$. niger autonomous with respect to D-Hiv feeding and allowed high level enniatin production.

Different cultivation protocols were run in this study to heterologously produce enniatin in strain DS3.1. The specific yields of enniatin accomplished in shake flask cultures were $0.04 \mathrm{~g} \cdot \mathrm{g}^{-1}$ dry weight biomass $(20 \mathrm{ml}$ and $1 \mathrm{l}$ cultures) and $0.18 \mathrm{~g} \cdot \mathrm{g}^{-1}$ dry weight biomass during the fed batch cultivation. In these cultivations, $5 \%$ glucose served as carbon source. The dynamics of enniatin $\mathrm{B}$ production clearly displayed primary metabolite kinetics in the fed batch run (Figure $7 \mathrm{~B}$ ), proving that the Tet-on system can ensure high level esyn1 expression during exponential growth. The highest specific enniatin yield, however, was observed for the batch cultivation $\left(0.29 \mathrm{~g} \cdot \mathrm{g}^{-1}\right.$ dry weight biomass), which used only $0.8 \%$ glucose as carbon source. Interestingly, the enniatin production followed secondary metabolite kinetics although the Tet-on system was switched on early during exponential growth phase (Figure 7A). Carbon-induced starvation has clearly been demonstrated to induce a plethora of secondary metabolites in $A$. niger $[9,50]$. It is thus conceivable that the transition to the post-exponential growth phase might have caused a metabolic shift in $A$. niger which favored secondary metabolite production in general and enniatin production in particular. The overall activated secondary metabolite machinery of $A$. niger might have strongly supported enniatin expression, e.g. by providing additional carbon and nitrogen due to autophagy [9], by ensuring a higher amino acid pool and/or by increasing the stability of the esyn1 transcript or the ESYN protein. Future studies are necessary to understand these processes; clearly, only systems-level insights will help to elucidate the molecular mechanisms behind.

\section{Conclusions}

This is the first report demonstrating that $A$. niger is a potent expression host for nonribosomal peptide synthetase. The strong inducibility of the Tet-on system combined with controlled bioreactor cultivation allowed the production of enniatin with yields which are high enough to become industrially relevant.

\section{Methods}

Strains, media and molecular techniques

Aspergillus strains used in this study are given in Table 1. Strains were grown on minimal medium (MM) [51] containing $1 \%\left(\mathrm{w} \cdot \mathrm{v}^{-1}\right)$ glucose and $0.1 \%\left(\mathrm{w} \cdot \mathrm{v}^{-1}\right)$ casamino acids or on complete medium (CM), containing $0.5 \%$ $\left(\mathrm{w} \bullet \mathrm{v}^{-1}\right)$ yeast extract in addition to MM. When required, 
Table 1 Strains used in this study

\begin{tabular}{|c|c|c|}
\hline Strain & Relevant genotype & Source \\
\hline \multicolumn{3}{|c|}{ Fusarium oxysporum } \\
\hline ETH1536 & Wild type & {$[54]$} \\
\hline \multicolumn{3}{|c|}{ Aspergillus niger } \\
\hline N402 & Wild type & {$[55]$} \\
\hline AB1.13 & pyrG $G^{-}, p_{t} T^{-}$ & {$[37]$} \\
\hline VG5.1 & $\begin{array}{l}\text { pyrG }{ }^{+}, \Delta k u s A \\
\text { (transformed with pVG2.2; single copy) }\end{array}$ & {$[15]$} \\
\hline DS3.1 & $\begin{array}{l}\text { pyrG }{ }^{+}, \text {prt } T^{-} \text {, esyn } 1 \\
\text { (transformed with pDS4.2, single copy) }\end{array}$ & This work \\
\hline ÖV4.3 & $\begin{array}{l}\text { pyrG }{ }^{+}, \text {prtT, esyn1, EkivR } \\
\text { (transformed with pÖV2.3; multi copy) }\end{array}$ & This work \\
\hline ÖV4.10 & $\begin{array}{l}\text { pyrG }{ }^{+}, \text {pyrtT } \text {, esyn1, EkivR } \\
\text { (transformed with pÖV2.3; multi copy) }\end{array}$ & This work \\
\hline ÖV4.11 & $\begin{array}{l}\text { pyrG }{ }^{+} \text {, pryt } T^{-} \text {, esyn1, EkivR } \\
\text { (transformed with pÖV2.3; multi copy) }\end{array}$ & This work \\
\hline
\end{tabular}

plates were supplemented with uridine $(10 \mathrm{mM})$. Transformation of $A$. niger and fungal chromosomal DNA isolation was performed as described [52]. All molecular techniques were carried out as described earlier [53].

The coding sequence of ESYN was PCR-amplified from a fosmid library of F. oxysporum ETH 1536 and ligated into the PmeI-linearized plasmid pVG2.2 (PgpdA:: rtTA::TcgrA-tetO7::Pmin::TtrpC-pyrG*, [15]). The resulting plasmid was named pDS4.2. The kivR gene from $F$. oxysporum ETH 1536 was PCR-amplified and ligated into the expression vector pNOM102 [56] via NcoI restriction. Thereby, the $ß$-glucuronidase gene was replaced by kivR. The resulting plasmid was named pÖV4.1. The proteasedeficient $A$. niger strain AB1.13 was transformed with pDS4.2 using its uracil-auxotrophy for selection [57]. Strain DS3.1 was co-transformed with plasmid pÖV4.1 and the selection plasmid p3SR2, which expresses acetamidase (amdS) as selection marker. Transformation of $A$. niger and fungal chromosomal DNA isolation was performed as described [52]. All molecular techniques were carried out as described earlier [53].

\section{Optimization of enniatin production}

Optimum cultivation conditions for enniatin production were identified using the statistical software program MODDE 9.1 (Umetrics). The screening experiments were performed in $20 \mathrm{ml}$ of $\mathrm{CM}$ which were inoculated with $5 \times 10^{6}$ spores $\cdot \mathrm{ml}^{-1}$ of strain DS3.1. All cultivations were performed at $250 \mathrm{rpm}$. After $16 \mathrm{~h}(\sim 1 \mathrm{~g}$ dry weight $\left.\cdot \mathrm{l}^{-1}\right)$, enniatin expression was induced by the addition of different concentrations of Dox. Cultures were harvested after $24 \mathrm{~h}$ by filtration and defined amounts of biomass and supernatant were used to isolate enniatin. Several cycles of optimization were performed which included the parameters cultivation time, concentration of inductor and D-Hiv, type of the cultivation medium, temperature, concentration of L-Val, L-Leu, and L-Ile, concentration of carbon source and talcum concentration (-350 mesh). As optimum cultivation condition was eventually identified: CM containing 5\% glucose and $10 \mathrm{~g} / \mathrm{l}$ talcum, temperature $26^{\circ} \mathrm{C}$, addition of $20 \mathrm{mM}$ D-Hiv, $20 \mathrm{mM} \mathrm{L}-\mathrm{Val}$ and $10 \mu \mathrm{g} / \mathrm{ml}$ Dox after $16 \mathrm{~h}$ of cultivation, total cultivation time $92 \mathrm{~h}$.

\section{Extraction and purification of enniatin}

Defined amounts of DS3.1 supernatant and biomass cultures were extracted with ethyl acetate. Extracts were centrifuged, dried over $\mathrm{Na}_{2} \mathrm{SO}_{4}$ and the solvent evaporated. Samples were dissolved in methanol, diluted if necessary and the enniatin concentration determined by HPLC-MS analysis. The HPLC-MS measurements for quantification were performed on an ESI-Triple-Quadrupol-MS, 6460 Series (Agilent Technologies) in multiple reaction monitoring mode. The utilized column was an Eclipse Plus C18, 2.1 $\times 50 \mathrm{~mm}$ column (Agilent Technologies) and the mobile phases were $\mathrm{H}_{2} \mathrm{O}+0.1 \%$ formic acid (A) and acetonitrile $+0.1 \%$ formic acid (B). The injection volume was set to $2 \mu \mathrm{l}$ and the flow rate was $0.3 \mathrm{ml} / \mathrm{min}$. The $\mathrm{m} / z$ value for the precursor ion was set to $640.4(\mathrm{~m} / z$ of [enniatin $\mathrm{B} \mathrm{H}^{+}$] - adduct) and for the fragment ions to 527.4 as quantifier, 427.3 and 196.2 as qualifier. For every set of measurements, a new calibration curve was made using enniatin isolated from $F$. oxysporum as an external standard. Peak areas were determined by manual integration using masshunter workstation quantitative analysis (Agilent).

For purifying enniatin by recrystallization, the crystals were resolved in a minimal amount of hot ethyl acetate. Acetonitrile was slowly added until clear crystals started to appear. The mother liquor was decanted and the crystals were washed several times with acetonitrile. Enniatin obtained from crystallization was applied to preparative HPLC (1100 series, Agilent Technologies) running isocratically $70 \%$ methanol, containing $0.1 \%$ formic acid on a C18-column (Grom-Sil 120 ODS-5 ST, $10 \mu \mathrm{m}, 250 \times 20$ mm, Grace).

\section{Identification and characterization of enniatin B}

${ }^{1} \mathrm{H}-\mathrm{NMR}$ and ${ }^{13} \mathrm{C}-\mathrm{NMR}$ spectra of enniatin $\mathrm{B}$ were recorded on a Bruker Avance 400 NMR-spectrometer. The signals of the non-deuterated solvent rests were used as standards. Chemical shifts are given in $\delta$-units (ppm) relative to the solvent signal. IR spectra were recorded on a Jasco FT-IR 4100 spectrometer. High-resolution massspectrometry (HRMS) using ESI-technique was performed on a LTQ Orbitrap XL apparatus. Data for the single-crystal structure determination of enniatin B were collected on an Oxford-Diffraction Xcalibur diffractometer, equipped with a CCD area detector Sapphire $S$ and a graphite monochromator utilizing $\operatorname{MoK}_{\alpha}$ radiation $(\lambda=0.71073 \AA$ ). 
Suitable crystals were attached to glass fibers using perfluoropolyalkylether oil and transferred to a goniostat where they were cooled to $150 \mathrm{~K}$ for data collection. The software packages used were CrysAlis CCD for data collection, CrysAlis Pro for cell refinement and data reduction.

\section{Bioreactor cultivation}

Submerged cultivations were performed with 6.6 liter BioFlo3000 bioreactors (New Brunswick Scientific, NJ, USA) and a detailed description of the fermentation settings was previously given 10 . In brief, glucose-limited batch cultivation was initiated by inoculation of $5 \mathrm{l}(\mathrm{kg})$ fermentation medium with conidial suspension of strain DS3.1 to give $10^{9}$ conidia $1^{-1}$. Glucose was sterilized separately from the fermentation medium and final concentration was $0.8 \%\left(\mathrm{w} \cdot \mathrm{v}^{-1}\right)$. Temperature of $30^{\circ} \mathrm{C}$ and $\mathrm{pH} 3$ were kept constant, the latter by computer controlled addition of $2 \mathrm{M} \mathrm{NaOH}$ or $1 \mathrm{M} \mathrm{HCl}$, respectively. Acidification of the culture broth was used as an indirect growth measurement [58]. When the culture reached the early exponential growth phase (corresponding to $1 \mathrm{~g}$ biomass dry weight $\left.\cdot \mathrm{kg}^{-1}\right)$, Dox $(10 \mu \mathrm{g} / \mathrm{ml})$, D-Hiv $(20 \mathrm{mM})$ and L-Val $(20 \mathrm{mM})$ were added.

The fed batch cultivation was started with 41 fermentation medium. Induction of the Tet-on system with Dox and addition of feeding medium (FM, $\left.0.046 \mathrm{l} \cdot \mathrm{h}^{-1}\right)$ was started when the culture reached the late exponential growth phase. FM is composed of fermentation medium with $5 \%$ glucose, $0.5 \% \mathrm{YE}, 0.1 \%$ casamino acids, $20 \mathrm{mM}$ D-Hiv and $20 \mathrm{mM} \mathrm{L}$-valine. Every $4-7 \mathrm{~h}, 10 \mu \mathrm{g} / \mathrm{ml}$ of Dox were added.

\section{Availability of supporting data}

The data sets supporting the results of this article are included within the article and its additional files.

\section{Additional files}

Additional file 1: Figure S1. Southern analysis of $A$. niger transformants. Additional file 2: Figure S2. COSY NMR-spectrum and crrystal structure of enniatin B.

Competing interests

The authors declare that they have no competing interests.

\section{Authors' contributions}

DS, ÖV, LR and FW performed the cloning experiments, SB, TK, ÖV and LR carried out the chemical analyses, TK and FW performed the batch and fed batch experiments. SB, DS, FW, LR, TK, ÖV, RS and VM designed the experiments and interpreted the results. FW, LR, RS and VM drafted the manuscript and were involved in writing the manuscript. All authors read and approved the final manuscript.

\section{Acknowledgements}

This project was partly funded by the Marie Curie International Training Network QuantFung (FP7-People-2013-ITN, Grant 607332) and supported by the Cluster of Excellence "Unifying Concepts in Catalysis" funded by the Deutsche Forschungsgemeinschaft DFG and coordinated by the TU Berlin.
Received: 26 June 2014 Accepted: 13 August 2014

Published online: 14 October 2014

\section{References}

1. Meyer V, Wu B, Ram AFJ: Aspergillus as a multi-purpose cell factory: current status and perspectives. Biotechnol Lett 2011, 33:469-476.

2. Lubertozzi D, Keasling JD: Developing Aspergillus as a host for heterologous expression. Biotechnol Adv 2009, 27:53-75.

3. Brakhage AA: Regulation of fungal secondary metabolism. Nat Rev Micro 2013, 11:21-32.

4. Brakhage AA, Schroeckh V: Fungal secondary metabolites - strategies to activate silent gene clusters. Fungal Genet Biol 2011, 48:15-22.

5. Wiemann P, Keller NP: Strategies for mining fungal natural products. J Ind Microbiol Biotechnol 2014, 41:301-313.

6. Amare MG, Keller NP: Molecular mechanisms of Aspergillus flavus secondary metabolism and development. Fungal Genet Biol 2014, 66:11-18.

7. Wiemann P, Guo CJ, Palmer JM, Sekonyela R, Wang CCC, Keller NP: Prototype of an intertwined secondary-metabolite supercluster. Proc Natl Acad Sci U S A 2013, 110:17065-17070.

8. Yin WB, Reinke AW, Szilágyi M, Emri T, Chiang YM, Keating AE, Pócsi I, Wang CCC, Keller NP: bZIP transcription factors affecting secondary metabolism, sexual development and stress responses in Aspergillus nidulans. Microbiology 2013, 159:77-88

9. Nitsche BM, Jørgensen TR, Akeroyd M, Meyer V, Ram AFJ: The carbon starvation response of Aspergillus niger during submerged cultivation: insights from the transcriptome and secretome. BMC Genomics 2012, 13:380.

10. Jørgensen TR, Nitsche BM, Lamers GE, Arentshorst M, van den Hondel CA, Ram AF: Transcriptomic insights into the physiology of Aspergillus niger approaching a specific growth rate of zero. Appl Environ Microbiol 2010, 76:5344-5355.

11. Nielsen MT, Nielsen JB, Anyaogu DC, Holm DK, Nielsen KF, Larsen TO, Mortensen UH: Heterologous reconstitution of the intact geodin gene cluster in Aspergillus nidulans through a simple and versatile PCR based approach. PLoS One 2013, 8:e72871.

12. Yaegashi J, Oakley BR, Wang CCC: Recent advances in genome mining of secondary metabolite biosynthetic gene clusters and the development of heterologous expression systems in Aspergillus nidulans. J Ind Microbiol Biotechnol 2014, 41:433-442.

13. Unkles SE, Valiante V, Mattern DJ, Brakhage AA: Synthetic biology tools for bioprospecting of natural products in eukaryotes. Chem Biol 2014, 21:502-508.

14. Chiang YM, Oakley CE, Ahuja M, Entwistle R, Schultz A, Chang SL, Sung CT, Wang CCC, Oakley BR: An efficient system for heterologous expression of secondary metabolite genes in Aspergillus nidulans. J Am Chem Soc 2013, 135:7720-7731.

15. Meyer V, Wanka F, van Gent J, Arentshorst M, van den Hondel CAMJJ, Ram AFJ: Fungal gene expression on demand: an inducible, tunable, and metabolism-independent expression system for Aspergillus niger. Appl Environ Microbiol 2011, 77:2975-2983.

16. Helmschrott C, Sasse A, Samantaray S, Krappmann S, Wagener J: Upgrading fungal gene expression on demand: improved systems for doxycycline-dependent silencing in Aspergillus fumigatus. Appl Environ Microbiol 2013, 79:1751-1754.

17. Wartenberg D, Vödisch M, Kniemeyer O, Albrecht-Eckardt D, Scherlach K Winkler R, Weide M, Brakhage AA: Proteome analysis of the farnesol-induced stress response in Aspergillus nidulans - The role of a putative dehydrin. J Proteomics 2012, 75:4038-4049.

18. Jørgensen TR, Park J, Arentshorst M, van Welzen AM, Lamers G, van Kuyk PA, Damveld RA, van den Hondel CAM, Nielsen KF, Frisvad JC, Ram AFJ: The molecular and genetic basis of conidial pigmentation in Aspergillus niger. Fungal Genet Biol 2011, 48:544-553.

19. Meyer V: Genetic engineering of filamentous fungi - progress, obstacles and future trends. Biotechnol Adv 2008, 26:177-185.

20. Pel HJ, de Winde JH, Archer DB, Dyer PS, Hofmann G, Schaap PJ, Turner G, de Vries RP, Albang R, Albermann $K$, Andersen MR, Bendtsen JD, Benen JAE, van den Berg M, Breestraat S, Caddick MX, Contreras R, Cornell M, Coutinho PM, Danchin EGJ, Debets AJM, Dekker P, van Dijck PWM, van Dijk A, Dijkhuizen L, Driessen AJM, d'Enfert C, Geysens S, Goosen C, Groot GSP, et al: Genome sequencing and analysis of the versatile cell factory Aspergillus niger CBS 513.88. Nat Biotechnol 2007, 25:221-231. 
21. Süssmuth R, Müller J, von Döhren H, Molnár I: Fungal cyclooligomer depsipeptides: from classical biochemistry to combinatorial biosynthesis. Nat Prod Rep 2011, 28:99-124.

22. Sy-Cordero AA, Pearce CJ, Oberlies NH: Revisiting the enniatins: a review of their isolation, biosynthesis, structure determination and biological activities. J Antibiot (Tokyo) 2012, 65:541-549.

23. Firáková S, Proksa B, Sturdíkova M: Biosynthesis and biological activity of enniatins. Pharmazie 2007, 62:563-568.

24. Krause $M$, Lindemann $A$, Glinski $M$, Hornbogen $T$, Bonse $G$, Jeschke $P$, Thielking G, Gau W, Kleinkauf H, Zocher R: Directed biosynthesis of new enniatins. J Antibiot (Tokyo) 2001, 54:797-864.

25. Feifel SC, Schmiederer T, Hornbogen T, Berg H, Süssmuth RD, Zocher R: In vitro synthesis of new enniatins: probing the alpha-D-hydroxy carboxylic acid binding pocket of the multienzyme enniatin synthetase. Chembiochem 2007, 8:1767-1770.

26. Gäumann E, Roth S, Etlinger L, Plattner PA, Nager U: Enniatin, ein neues, gegen Mykobaktieren wirksames Antibiotikum. Experientia 1947, III:202-203.

27. Jayasinghe L, Abbas HK, Jacob MR, Herath WHMW, Nanayakkara NPD: N-Methyl-4-hydroxy-2-pyridinone analogues from Fusarium oxysporum. J Nat Prod 2006, 69:439-442.

28. Mckee TC, Bokesch HR, McCormick JL, Rashid MA, Spielvogel D, Gustafson KR, Alavanja MM, Cardellina JH, Boyd MR: Isolation and characterization of new anti-HIV and cytotoxic leads from plants, marine, and microbial organisms. J Nat Prod 1997, 60:431-438.

29. Dornetshuber R, Heffeter P, Kamyar MR, Peterbauer T, Berger W, LemmensGruber R: Enniatin exerts p53-dependent cytostatic and p53independent cytotoxic activities against human cancer cells. Chem Res Toxicol 2007, 20:465-473.

30. Herrmann M, Zocher R, Haese A: Effect of disruption of the enniatin synthetase gene of virulence of Fusarium avenaceum. Am Phytopathol SoC 1996, 9:226-232.

31. Levy D, Bluzat A, Seigneuret M, Rigaud JL: Alkali cation transport through liposomes by the antimicrobial fusafungine and its constitutive enniatins. Biochem Pharmacol 1995, 50:2105-2107.

32. Ovchinnikow YA, Ivanov VT, Evstratov AV, Mikhaleva II, Bystrov VF, Portnova SL, Balashova T, Meshcheryakova E, Tulchinsky VM: The enniatin ionophores. Conformation and ion binding properties. Int J Pept Protein 1974, 6:465-498.

33. Kamyar M, Rawnduzi P, Studenik CR, Kouri K, Lemmens-Gruber R: Investigation of the electrophysiological properties of enniatins. Arch Biochem Biophys 2004, 429:215-223

34. Tonshin AA, Teplova W, Andersson MA, Salkinoja-Salonen MS: The Fusarium mycotoxins enniatins and beauvericin cause mitochondrial dysfunction by affecting the mitochondrial volume regulation, oxidative phosphorylation and ion homeostasis. Toxicology 2010, 276:49-57.

35. Hiraga K, Yamamoto S, Fukuda H, Hamanaka N, Oda K: Enniatin has a new function as an inhibitor of Pdr5p, one of the $A B C$ transporters in Saccharomyces cerevisiae. Biochem Biophys Res Commun 2005, 328:1119-1125.

36. Miyazaki A, Kanome T, Watanabe T: Inhibitors of acyl-coenzyme a: cholesterol acyltransferase. Curr Drug Targets Cardiovasc Haematol Disord 2005, 5:463-469.

37. Mattern IE, van Noort JM, van den Berg P, Archer DB, Roberts IN, van den Hondel CA: Isolation and characterization of mutants of Aspergillus niger deficient in extracellular proteases. Mol Gen Genet 1992, 234:332-336.

38. Madry N, Zocher R, Kleinkauf H: Enniatin Production by Fusarium oxysporum in chemically defined media. Appl Microbiol Biotechnol 1983, 17:75-79.

39. Driouch H, Sommer B, Wittmann C: Morphology engineering of Aspergillus niger for improved enzyme production. Biotechnol Bioeng 2010, 105:1058-1068.

40. Zhukhlistova NE: X-ray crystal structure of the complex of enniatin B with KNCS. Crystallogr Rep 2002, 47:433-442.

41. Burmeister HR, Plattner RD: Enniatin production by Fusarium tricinctum and its effect on germinating wheat seeds. Phytopathology 1987, 77:1483-1487.

42. Jørgensen TR, Goosen T, Hondel CAMJJ Van D, Ram AFJ, Iversen J L: Transcriptomic comparison of Aspergillus niger growing on two different sugars reveals coordinated regulation of the secretory pathway. BMC Genomics 2009, 10:44
43. Hrdlicka PJ, Sørensen AB, Poulsen BR, Ruijter GJG, Visser J, Iversen JJL: Characterization of nerolidol biotransformation based on indirect on-line estimation of biomass concentration and physiological state in batch cultures of Aspergillus niger. Biotechnol Prog 2004, 20:368-376.

44. Matthes D, Richter L, Müller J, Denisiuk A, Feifel SC, Xu Y, Espinosa-Artiles P, Süssmuth RD, Molnár I: In vitro chemoenzymatic and in vivo biocatalytic syntheses of new beauvericin analogues. Chem Commun (Camb) 2012, 48:5674-5676.

45. Yu D, Xu F, Zi J, Wang S, Gage D, Zeng J, Zhan J: Engineered production of fungal anticancer cyclooligomer depsipeptides in Saccharomyces cerevisiae. Metab Eng 2013, 18:60-68.

46. Hu DX, Bielitza M, Koos P, Ley SV: A total synthesis of the ammonium ionophore, (-)-enniatin B. Tetrahedron Lett 2012, 53:4077-4079.

47. Audhya TK, Russell DW: Spectrophotometric determination of enniatin a and valinomycin in fungal extracts by ion complexation. Anal Lett 1973, 6:265-274.

48. Audhya TK, Russell DW: Production of enniatin A. Can J Microbiol 1973, 19:1051-1054

49. Kwon MJ, Jørgensen TR, Nitsche BM, Arentshorst M, Park J, Ram AFJ, Meyer V: The transcriptomic fingerprint of glucoamylase over-expression in Aspergillus niger. BMC Genomics 2012, 13:701.

50. King HL, Dyar RE, Wilken R: Ketopantoyl lactone and acid reductases. J Biol Chem 1974, 249:4689-4695.

51. Bennett JW, Lasure LL: More Gene Manipulations in Fungi. New York Acad Press Inc. San Diego, California; 1991:441-457.

52. Meyer V, Ram A, Punt P: Genetics, Genetic Manipulation and Approaches to Strain Improvement of Filamentous Fungi. In Man Ind Microbiol Biotechnol. 3rd edition. NY: Wiley; 2010:318-329

53. Sambrook J, Russell DW: Molecular Cloning: A Laboratory Manual. New York Cold Spring Habour Lab Press New York; 2001:2344.

54. Zocher R, Keller U, Kleinkauf H: Enniatin synthetase, a novel type of multifunctional enzyme catalyzing depsipeptide synthesis in Fusarium oxysporum. Biochemistry 1982, 21:43-48.

55. Bos CJ, Debets AJM, Swart K, Huybers A, Kobus G, Slakhorst SM: Genetic analysis and the construction of master strains for assignment of genes to six linkage groups in Aspergillus niger. Curr Genet 1988, 14:437-443.

56. Punt PJ, Dingemanse MA, Kuyvenhoven A, Soede RDM, Pouwels PH, van den Hondel CAMJJ: Functional elements in the promoter region of the Aspergillus nidulans gpdA gene encoding glyceraldehyde-3-phosphate dehydrogenase. Gene 1990, 93:101-109.

57. Punt PJ, van den Hondel CA: Transformation of filamentous fungi based on hygromycin B and phleomycin resistance markers. Methods Enzymol 1992, 216:447-457.

58. Iversen JJL, Thomsen JK, Cox RP: On-line growth measurements in bioreactors bytitrating metabolic proton exchange. Appl Environ Microbiol 1994, 42:256-262

doi:10.1186/s40694-014-0004-9

Cite this article as: Richter et al:: Engineering of Aspergillus niger for the production of secondary metabolites. Fungal Biology and Biotechnology 2014 1:4.

\section{Submit your next manuscript to BioMed Central and take full advantage of:}

- Convenient online submission

- Thorough peer review

- No space constraints or color figure charges

- Immediate publication on acceptance

- Inclusion in PubMed, CAS, Scopus and Google Scholar

- Research which is freely available for redistribution 